\title{
Schauder bases and reflexivity
}

N. J. KALTON (Cambridge and Lehigh)

1. Introduction. In [11] Singer showed that a Banach space in which every basic sequence is boundedly-complete or every basic sequence is shrinking is reflexive; and in [12] Zippin extended this result by replacing basic sequence by basis. In this paper I shall obtain extensions of both Singer's result and Zippin's result to cover more general locally convex spaces (also improving on the result of Retherford [9]).

Suppose $E$ is a locally convex space with a Schauder basis $\left(x_{n}\right)$; then $\left(f_{n}\right)$ will always denote the dual sequence in $E^{\prime} ; P_{n}$ will denote the map

$$
P_{n} x=\sum_{i=1}^{n} f_{i}(x) x_{i}
$$

and $P_{n}^{\prime}$ will be the dual map

$$
P_{n}^{\prime} f=\sum_{i=1}^{n} f\left(x_{i}\right) f_{i}
$$

in $E^{\prime}$. The subspace of $E^{\prime}$ consisting of all $f$ such that $\lim P_{n}^{\prime} f=f$ in the strong topology will be denoted by $H$; the topology on $H$ will be assumed to be the strong topology $\beta\left(E^{\prime}, E\right)$.

The Schauder basis $\left(x_{n}\right)$ is said to be shrinking if $H=E^{\prime}$ (i.e. $\left(f_{n}\right)$ is a Schauder basis for $E^{\prime}$ in the strong topology); it is said to be $\gamma$-complete, or boundedly-complete, if whenever $\left(\sum_{i=1}^{n} \alpha_{i} x_{i}\right)_{n=1}^{\infty}$ is bounded, then $\sum_{i=1}^{\infty} a_{i} x_{i}$ converges. It is shown in [13] that $E$ is semi-reflexive if and only if $\left(x_{n}\right)$ is $\gamma$-complete and shrinking.

The basis $\left(x_{n}\right)$ is regular (see [6]) if there exists a neighbourhood $V$ of zero such that $x_{n} \notin V$ for all $n$. A bounded regular basis is called normalised. Finally, $\left(x_{n}\right)$ is simple if for all $f \in E^{\prime}\left(P_{n}^{\prime} f\right)_{n=1}^{\infty}$ is strongly bounded (see [5]); if $\left(x_{n}\right)$ is simple, $\left(P_{n}^{\prime}\right)$ is strongly equicontinuous.

In addition I shall use the following definitions: 
Definition 1.1. $\left(x_{n}\right)$ is equi-regular if $\left(f_{n}\right)$ is equicontinuotis; $\left(x_{n}\right)$ is equi-normalised if it is equi-regular and bounded.

LEMnNa 1.2. If $\left(x_{n}\right)$ is simple and regular, and $E$ is quasi-barrelled, then $\left(x_{n}\right)$ is equi-regular.

As $\left(P_{n}^{\prime}\right)_{n=1}^{\infty}$ is equicontinuous, if $A$ is bounded in $E$, then $\bigcup_{n=1}^{\infty} P_{n}(A)$ is bounded and hence so is $\left\{f_{n}(a) x_{n} ; a \in A, n=1,2, \ldots\right\}$; as $\left(x_{n}\right)$ is regular

$$
\sup _{n} \sup _{a \in A}\left|f_{n}(a)\right|<\infty
$$

i.e. $\left(f_{n}\right)$ is strongly bounded and hence equicontinuous.

If $\left(x_{n}\right)$ is equi-normalised, then $\left(f_{n}\right)$ is equi-normalised as a Schauder basis of $H$.

2. Sequences of type $P$ and $P^{*}$. Bases and basic sequences of types $P$ and $P^{*}$ were introduced and studied by Singer [11]; later the definition was extended by Dubinsky and Retherford to generally locally convex spaces [1]. For convenience I shall extend the definition to biorthogonal systems; if $\left(x_{n}\right)$ is a sequence in $E$, and $\left(f_{n}\right)$ a sequence in $E^{\prime}$ with $f_{n}\left(x_{m}\right)=\delta_{n m}$ (Kronecker delta), then $\left(x_{n} ; f_{n}\right)$ is a biorthogonal system in $E$.

Definition 2.1. $\left(x_{n} ; f_{n}\right)$ is of type $P$ if there exists a neighbourhood $V$ of zero with $x_{n} \notin V$ for all $n$, and the sequence $\left(\sum_{i=1}^{n} x_{i} ; n=1,2, \ldots\right)$
is bounded.

Definition 2.2. $\left(x_{n} ; f_{n}\right)$ is of type $P^{*}$ (in $E$ ) if $\left(x_{n}\right)$ is bounded and $\left(\sum_{i=1}^{n} f_{i} ; n=1,2, \ldots\right)$ is strongly bounded in $E^{\prime}$.

If $G$ is a subspace of $E$ with $x_{n} \in G$ for all $n$, and $g_{n}$ is the restriction of $f_{n}$ to $G$, then $\left(x_{n} ; g_{n}\right)$ forms another biorthogonal system; if $\left(x_{n} ; f_{n}\right)$ is of type $P^{*}$, then so is $\left(x_{n} ; g_{n}\right)$, but the converse is not necessarily true.

If $\left(x_{n}\right)$ is a Schauder basis of $E$, then $\left(x_{n} ; f_{n}\right)$ forms a biorthogonal system, and so I shall say $\left(x_{n}\right)$ is type $P$ or $P^{*}$ according as $\left(x_{n} ; f_{n}\right)$ is type $P$ or $P^{*}$. If $\left(n_{j}\right)$ is an increasing sequence of positive integers such that $\left(x_{n_{j}} ; f_{n_{j}}\right)$ is of type $P$ or $P^{*}$, then I shall say that $\left(x_{n_{j}}\right)$ is a type $P$ or $P^{*}$ subsequence of $\left(x_{n}\right)$; this may not be the same as stating that $\left(x_{n_{j}}\right)$ is a type $P$ or $P^{*}$ basis of the subspace $\overline{l i n}\left(x_{n_{f}}\right)_{j=1}^{\infty}$.

With some restrictions the same duality results as proved by Singer [11] for the Banach space case hold. The proof of the next result is obvious. Proposition 2.3. If $\left(x_{n}\right)$ is a type $P^{*}$ Schauder basis of $E$, then $\left(f_{n}\right)$ is a type $P$ Schauder basis of $H$.

For convenience I shall call a space $E$ in which every bounded set is strongly (i.e. $\beta\left(E, E^{\prime}\right)$ ) bounded, a $W$-space. $E$ is a $W$-space if and only if its weak dual $\left\{E^{\prime}, \sigma\left(E^{\prime}, E\right)\right\}$ is a $W$-space; any sequentially complete space is a $W$-space. Thus any semi-reflexive or barrelled space is a $W$-space. Any Schauder basis of a $W$-space is simple.

Proposition 2.4. Let E be a W-space, and let $\left(x_{n}\right)$ be a Schauder basis of $E$. If $\left(x_{n}\right)$ is of type $P$, then $\left(f_{n}\right)$ is a type $P^{*}$ basis of $H$; if $\left(f_{n}\right)$ is a type $P$ basis of $H$, then $\left(x_{n}\right)$ is of type $P^{*}$.

$\left(x_{n}\right)$ is certainly simple, and hence if $\left(f_{n}\right)$ is of type $P$, then by Proposition 3.1 of [6], $\left(x_{n}\right)$ is bounded and hence of type $P^{*}$.

If $\left(x_{n}\right)$ is of type $P$, then $\left(x_{n}\right)$ is regular; if $x \in E$, then $x=\sum_{i=1}^{\infty} f_{i}(x) x_{i}$ so that $\lim _{i \rightarrow \infty} f_{i}(x)=0$. Thus $\left(f_{n}\right)$ is weakly bounded and hence strongly bounded. Let $s_{n}=\sum_{i=1}^{n} x_{i}$; then $\left(s_{n}\right)$ is bounded and hence $\beta\left(E, E^{\prime}\right)$ bounded. The natural injection $E \rightarrow H^{\prime}$ is continuous for the strong topologies $\beta\left(E, E^{\prime}\right)$ and $\beta\left(H^{\prime}, H\right)$ so that $\left(s_{n}\right)$ is $\beta\left(H^{\prime}, H\right)$ bounded (when $E$ is regarded as a subspace of $\left.H^{\prime}\right)$. Thus $\left(f_{n}\right)$ is of type $P^{*}$ for $H$.

Proposition 2.5. If every strongly bounded sequence in $E^{\prime}$ is equicontinuous and $\left(x_{n}\right)$ is a simple Schauder basis of $E$, such that $\left(f_{n}\right)$ is a type $P^{*}$ basis of $H$, then $\left(x_{n}\right)$ is a type $P$ basis of $E$.

Again considering $E$ as a subspace of $H^{\prime}$, the sequence $s_{n}=\sum_{i=1}^{n} x_{i}$ is $\beta\left(H^{\prime}, H\right)$ bounded; applying Proposition 5.3 of [5], $\left(s_{n}\right)$ is bounded in the original topology on $E$. Also $\left(f_{n}\right)$ is strongly bounded and hence equicontinuous; thus $\left(x_{n}\right)$ is regular and so of type $P$.

The condition that every strongly bounded sequence in $E^{\prime}$ is equicontinuous appears in several results of Dubinsky and Retherford [1]; this class includes all quasi-barrelled or $D F$-spaces. However, it is not quite as wide as it might appear.

Proposition 2.6. Suppose every strongly bounded sequence in $E^{\prime}$ is equicontinuous, and $\left(x_{n}\right)$ is a Schauder basis of $E$; then:

(i) $E$ is quasi-barrelled in its Mackey topology, $\tau\left(E, E^{\prime}\right)$.

(ii) If $E$ is a sequentially complete DF-space, $E$ is barrelled.

(iii) If $\left(x_{n}\right)$ is simple, then $\left(x_{n}\right)$ is a Schauder basis of $\left\{E, \tau\left(E, E^{\prime}\right)\right\}$.

(i) Suppose $A \subset E^{\prime}$ is absolutely convex, strongly bounded and weakly closed; then any countable subset of $A$ is weakly relatively compact. By Theorem 6 of [2], as $\left(E^{\prime}, \sigma\left(E^{\prime}, E\right)\right)$ may be regarded as a sequence space, $A$ is weakly compact. Thus $A$ is Mackey equicontinuous and so $E$ is quasi-barrelled.

(ii) A separable $D F$-space has a Mackey topology (see [3]).

(iii) If. $\left(x_{n}\right)$ is simple; then a slight modification of Corollary 4 of Proposition 5.3 of [5] gives the result.

Studia Mathematica XxxvIII 
3. Bases with subsequences of types $P$ and $P^{*}$. In [11], Singer showed that from a basic sequence of type $P$ in a Banach space one can obtain a basic sequence of type $P^{*}$ and vice versa. Here I shall obtain similar results for bases of locally convex spaces with subsequences of types $P$ or $P^{*}$ (see above, section 2). Suppose $\left(x_{n}\right)$ is a Schauder basis of $E$ with a subsequence $\left(x_{n_{j}}\right)$ of type $P$ (where $\left(n_{j}\right)$ is an increasing sequence of positive integers); let $\left(s_{n}\right)$ be defined by

$$
s_{k}= \begin{cases}x_{k} & \text { if } k \neq n_{j} \text { for all } j \\ \sum_{i=1}^{j} x_{n_{i}} & \text { if } k=n_{j} .\end{cases}
$$

THEOREM 3.1. $\left(s_{n}\right)$ is a Schauder basis of $E$; if $\left(x_{n}\right)$ is simple, then $\left(s_{n_{j}}\right)$ is a type $P^{*}$ subsequence of $\left(s_{n}\right)$.

Let

$$
u_{k}= \begin{cases}f_{k} & \text { if } k \neq n_{j} \\ f_{n_{j}}-f_{n_{j+1}} & \text { if } k=n_{j}\end{cases}
$$

Then, if $n_{j}<m \leqslant n_{j+1}$,

$$
\begin{aligned}
\sum_{k=1}^{m} f_{k}(x) x_{k}-\sum_{k=1}^{m} u_{k}(x) s_{k} & =\sum_{i=1}^{j} f_{n_{i}}(x) x_{n_{i}}-\sum_{i=1}^{j} u_{n_{i}}(x) s_{n_{i}} \\
& =f_{n_{j+1}}(x) s_{n_{j}} .
\end{aligned}
$$
that

As $\left(x_{n_{j}}\right)$ is regular, $\lim _{j \rightarrow \infty} f_{n_{j+1}}(x)=0$; and as $\left(s_{n_{j}}\right)$ is bounded it follows

$$
x=\sum_{k=1}^{\infty} f_{k}(x) x_{k}=\sum_{k=1}^{\infty} u_{k}(x) s_{k},
$$

so that $\left(s_{k}\right)$ is a Schauder basis of $E$ (with dual $\left(u_{k}\right)$ ).

If $\left(x_{n}\right)$ is simple, and $A \subset E$ is bounded, the set $\left\{P_{n}(a) ; a \in A\right.$, $n=1,2, \ldots\}$ is bounded (see [5]) and so $\left\{f_{n_{j}}(a) x_{n_{j}} ; a \in A, j=1,2, \ldots\right\}$ is bounded. As $\left(x_{n_{j}}\right)$ is regular,

$$
\sup _{a \in A} \sup _{j}\left|f_{n_{j}}(a)\right|<\infty
$$

so that $\left(f_{n_{j}}\right)$ is strongly bounded.

However

$$
\sum_{i=1}^{j} u_{n_{i}}=f_{n_{1}}-f_{n_{j+1}}
$$

and so $\left(s_{n_{j}}\right)$ is a type $P^{*}$ subsequence of $\left(s_{n}\right)$. Furthermore, as $\lim _{j \rightarrow \infty} f_{n_{j}}=0$ weakly, $\sum_{i=1}^{\infty} u_{n_{i}}$ converges weakly.
Now suppose $\left(s_{n}\right)$ is a Schauder basis of $E$ (with dual $\left(u_{n}\right)$ ) and that $\left(n_{j}\right)$ is an increasing sequence of integers. Let

$$
x_{k}= \begin{cases}s_{k} & \text { if } k \neq n_{j} \text { for all } j, \\ s_{n_{j}}-s_{n_{j-1}} & \text { if } k=n_{j} \text { (letting } s_{n_{0}}=0 \text { ). }\end{cases}
$$

THEOREM 3.2. (i) If $\left(s_{n_{j}}\right)$ is bounded and $\sum_{j=1}^{\infty} u_{n_{j}}$ converges weakly, then $\left(x_{n}\right)$ is a Schauder basis of $E$.

(ii) If $\left(s_{n_{j}}\right)$ is a type $P^{*}$ subsequence of $\left(s_{n}\right)$ and $\left(\sum_{j=1}^{k} u_{n_{j}}\right)_{k=1}^{\infty}$ is equicontinuous, then $\left(x_{n}\right)$ is a Schauder basis of $E$ with $\left(x_{n_{j}}\right)$ a subsequence of type $P$ (i) Let

$$
f_{k}= \begin{cases}u_{k} & \text { if } k \neq n_{j} \\ \sum_{i=j}^{\infty} u_{n_{i}} \text { if } k=n_{j} .\end{cases}
$$

Then !

$$
\sum_{i=1}^{k} f_{i}(x) x_{i}-\sum_{i=1}^{k} u_{i}(x) s_{i}=f_{n_{j+1}}(x) s_{n_{j}}, \quad \text { where } n_{j}<k \leqslant n_{j+1} .
$$

As $\left(s_{n_{j}}\right)$ is bounded, and $\lim _{j \rightarrow \infty} f_{n_{j+1}}(x)=0,\left(x_{n}\right)$ is a Schauder basis of $E$.

(ii) The sequence $\left\{\sum_{i=1}^{k} u_{n_{i}}^{j \rightarrow \infty} ; k=1,2, \ldots\right\}$ has a weak cluster point $v$; obviously $v\left(s_{n_{i}}\right)=1$ for all $i$ and $v\left(s_{k}\right)=0$ for all $k \neq n_{i}$. Thus $v=\sum_{i=1}^{\infty} u_{n_{i}}$
and, by $(\mathrm{i}),\left(x_{n}\right)$ is a Schauder basis of $E$ with

$$
f_{n_{j}}=v-\sum_{i=1}^{j-1} u_{n_{i}}
$$

so that $\left(f_{n_{j}}\right)$ is equicontinuous. Therefore $\left(x_{n_{j}}\right)$ is regular and hence of type $P$. basis.

TheOREM 3.3. Let $E$ be sequentially complete and possess a schauder

(i) If every Schauder basio sequence in $E$ is $\gamma$-complete, then $E$ is semireflexive.

(ii) If every Schauder basic sequence in $E$ is shrinking, then $E$ is semireflexive.

(i) Suppose $E$ is not semi-reflexive and $\left(x_{n}\right)$ is a Schauder basis of $E$; then by Theorem 3.3 of [5], $\left(x_{n}\right)$ is not shrinking. As $E$ is sequentially complete, $\left(x_{n}\right)$ is simple, and so by Theorem 5.4 of [6] there exists a block basic sequence $\left(y_{n}\right)$ which is bounded and $f \in E^{\prime}$ such that $f\left(y_{n}\right)=1 .\left(\left(y_{n}\right)\right.$ is a block basic sequence if it takes the form

$$
y_{j}=\sum_{n_{j-1}+1}^{n_{j}} \lambda_{i} x_{i}
$$


where $n_{0}=0$ and $\left(n_{j}\right)$ is an increasing sequence).

Let $F=\varlimsup$ in $\left(y_{j}\right)$; then $F$ is sequentially complete and $\left(y_{j}\right)$ is a simple Schauder basis of $F$. If $g$ denotes the restriction of $f$ to $F$, then $g=\sum_{i=1}^{\infty} g_{i}$ weakly, where $\left(g_{j}\right)$ is dual to $\left(y_{j}\right)$ in $F^{\prime}$. By Theorem 3.2 , if $z_{j}=y_{j}-y_{j-1}$ (with $y_{0}=0$ ), then $\left(z_{j}\right)$ is a Schauder basis of $F$; hence $\left(z_{j}\right)$ is $\gamma$-complete. Thus $\sum_{j=1}^{\infty} z_{j}$ converges; but

$$
f_{n}\left(\sum_{j=1}^{\infty} z_{j}\right)=\lim _{k \rightarrow \infty} f_{n}\left(y_{k}\right)=0 \quad \text { for all } n .
$$

Therefore $\sum_{j=1}^{\infty} z_{j}=0$, but $f\left(\sum_{j=1}^{\infty} z_{j}\right)=1$; this is the required contradiction.

(ii) Again suppose $\left(x_{n}\right)$ is a Schauder basis of $E$ and that $E$ is not semireflexive; then $\left(x_{n}\right)$ is not $\gamma$-complete. There exists a sequence $\left(\lambda_{n}\right)$ of scalars such that $\left(\sum_{i=1}^{k} \lambda_{i} x_{i}\right)_{k=1}$ is bounded but does not converge; as $E$ is sequentially complete, there exists an increasing sequence of integers $n_{j}$ with $n_{0}=0$ such that if

$$
y_{j}=\sum_{n_{j-1}+1}^{n_{j}} \lambda_{i} x_{i},
$$

then $\left(y_{j}\right)$ is a block basic sequence of type $P$. Since $F=\overline{l n}\left(y_{n}\right)$ is sequentially complete, $\left(y_{n}\right)$ is a simple Schauder basis of $F$, and, by Theorem 3.1, there exists a Schauder basis of $F,\left(z_{n}\right)$, of type $P^{*}$. If $\left(h_{n}\right)$ is the dual sequence of $\left(z_{n}\right)$ in $F^{\prime}$, then, as shown in the proof of Theorem 3.1, $\sum_{i=1}^{\infty} h_{i}$ converges weakly. Thus as $\left(z_{n}\right)$ is shrinking $\sum_{i=1}^{\infty} h_{i}$ converges strongly, and so $\lim _{n \rightarrow \infty} h_{n}=0$ strongly. However, $\left(z_{n}\right)$ is bounded, and

$$
\sup _{m}\left|h_{n}\left(z_{m}\right)\right|=1 \quad \text { for all } n
$$

and this is again a contradiction.

This theorem is a considerable improvement on the results of Retherford [9], who obtained the same result under the extra conditions that $E$ is barrelled and every closed subspace of $E$ with a Schauder basis is barrelled.

4. Semi-shrinking and semi- $\gamma$-complete bases. In [8], Pełczyński and Szlenk introduced semi-shrinking and semi-boundedly-complete bases of Banach spaces; these definitions generalise naturally to locally convex spaces.
Definition 4.1. A Schauder basis $\left(x_{n}\right)$ is semi-shrinking if $\left(x_{n}\right)$ is regular and $\lim _{n \rightarrow \infty} x_{n}=0$ weakly.

$$
\lim _{n \rightarrow \infty}
$$

Definition 4.2. A Schauder basis $\left(x_{n}\right)$ is semi- $\gamma$-complete if $\left(x_{n}\right)$ is bounded and whenever $\left(\sum_{i=1}^{n} \lambda_{i} x_{i}\right)_{n=1}^{\infty}$ is bounded, then $\lim _{n \rightarrow \infty} \lambda_{n}=0$.

A semi-shrinking basis is normalised, but the same is not true for a semi- $\gamma$-complete basis (e.g. the standard basis of $l^{2}$ in its weak topology). A normalised shrinking basis is semi-shrinking and a normalised $\gamma$-complete basis is semi- $\gamma$-complete, but the converses are false (see [8] and [10]).

Proposision 4.3. Let $\left(x_{n}\right)$ be a simple Schauder basis. of $E$.

(i) If $\left(x_{n}\right)$ is semi- $\gamma$-complete, then $\left(f_{n}\right)$ is a semi-shrinking basis of $H$.

(ii) If every strongly bounded sequence in $E^{\prime}$ is equicontinuous and $\left(x_{n}\right)$ is semi-shrinking, then $\left(f_{n}\right)$ is semi- $\gamma$-complete for $H$.

(i) Since $\left(x_{n}\right)$ is bounded, $\left(f_{n}\right)$ is regular in the strong topology. Suppose $\chi \epsilon\left(E^{\prime}, \beta\left(E^{\prime}, E\right)\right)^{\prime}$; for $f \epsilon E^{\prime}$, as $\left(x_{n}\right)$ is simple

Therefore

$$
\sup _{n}\left|\chi\left(\sum_{i=1}^{n} f\left(x_{i}\right) f_{i}\right)\right|<\infty \text {. }
$$

$$
\sup _{n}\left|\sum_{i=1}^{n} f\left(x_{i}\right) \chi\left(f_{i}\right)\right|<\infty,
$$

so $\left(\sum_{i=1}^{n} \chi\left(f_{i}\right) x_{i} ; n=1,2, \ldots\right)$ is bounded in $E$; by assumption $\lim _{n \rightarrow \infty} \chi\left(f_{n}\right)=0$, and so $\lim _{n \rightarrow \infty} f_{n}=0$ in $\sigma\left(E^{\prime}, E^{\prime \prime}\right)$.

(ii) By Theorem 3.4 of $[6],\left(f_{n}\right)$ is strongly normalised. Suppose $\left\{\sum_{i=1}^{n} \lambda_{i} f_{i} ; n=1,2, \ldots\right\}$ is strongly bounded; then this set is weakly compact and so possesses a cluster point $f$, where $f\left(x_{i}\right)=\lambda_{i}$. Thus

$$
\lim _{n \rightarrow \infty} \lambda_{n}=\lim _{n \rightarrow \infty} f\left(x_{n}\right)=0 \text {. }
$$

In [7], Pełczyński and Singer introduced a very useful method of obtaining a new basis from a given basis of a Banach space, called the method of block perturbation. Under certain circumstances this may be extended to apply to locally convex spaces. Let $\left(x_{n}\right)$ be a simple regular Schauder basis of $E$ and let $u_{j}$ be a bounded block basic sequence,

$$
u_{j}=\sum_{m_{j-1}+1}^{m_{j}} \alpha_{i} x_{i}
$$


(where $m_{0}=0$ and $\left(m_{j}\right)$ is an increasing sequence of integers). Suppose that there exists a sequence $\left\{p_{j}\right\}$ with $m_{j-1}<p_{j} \leqslant m_{j}$ such that $\alpha_{p_{j}}=0$ for all $j$; then the sequence

$$
y_{n}=x_{n}+\sum_{k=1}^{\infty} \delta_{n p_{k}} u_{k}
$$

is a block perturbation of $\left(x_{n}\right)$.

LEMMA 4.4. $\left(y_{n}\right)$ is a simple regular Schauder basis of $E$; if $\left(x_{n}\right)$ is equi-regular, then so is $\left(y_{n}\right)$.

Let $g_{n}=f_{n}-\alpha_{n} f_{t_{n}}$, where $t_{n}=p_{l k}$ for $m_{k-1}<n \leqslant m_{l k}$. Then $g_{n}\left(y_{m}\right)=\delta_{n m}$ and as in [7]

$$
\sum_{n=1}^{N} g_{n}(x) y_{n}-\sum_{n=1}^{N} f_{n}(x) x_{n}=f_{p_{r}}(x)\left(\beta_{N} u_{r}-P_{N} u_{r}\right)
$$

where $m_{r-1}<N \leqslant m_{r}$ and $\beta_{N}=0$ if $N<p_{r}$ and $\beta_{N}{ }^{*}=1$ if $p_{r} \leqslant N$.

As $\left(x_{n}\right)$ is simple, the set $\left(\beta_{N} u_{r}-P_{N} u_{r}\right)_{N=1}^{\infty}$ is bounded, and $\left(x_{n}\right)$ is regular $\lim _{N \rightarrow \infty} f_{p_{r}}(x)=0$. Thus

$$
\sum_{i=1}^{\infty} g_{i}(x) y_{i}=x
$$

and $\left(y_{n}\right)$ is a Schauder basis of $E$.

$$
\sum_{n=1}^{N} f\left(y_{n}\right) g_{n}-\sum_{n=1}^{N} f\left(x_{n}\right) f_{n}=f\left(\beta_{N} u_{r}-P_{N} u_{r}\right) f_{p_{r}}
$$

and as $\left(x_{n}\right)$ is simple and regular, it follows that $\left(f_{p_{r}}\right)$ is strongly bounded; thus the set $\left(\sum_{n=1}^{N} f\left(y_{n}\right) g_{n} ; N=1,2, \ldots\right)$ is strongly bounded, i.e. $\left(y_{n}\right)$ is
simple.

If $\left(f_{n}\right)$ is equicontinuous, then as $\left(\alpha_{n}\right)$ is bounded (since $\left(x_{n}\right)$ is regular and simple), $g_{n}=f_{n}-\alpha_{n} f_{t_{n}}$ is equicontinuous.

THEOREM 4.5. Let $\left(x_{n}\right)$ be a simple regular Schauder basis of $E$; if every block perturbation of $\left(x_{n}\right)$ is semi-shrinking, then $\left(x_{n}\right)$ is shrinking.

By Theorem 5.4 of [6], if $\left(x_{n}\right)$ is not shrinking, there exists a bounded block basic sequence $\left(y_{j}\right)$ and $f \in E^{\prime}$ with $f\left(y_{j}\right)=1$ for all $j$. Let

$$
y_{j}=\sum_{n_{j-1}+1}^{n_{j}} \alpha_{i} x_{i},
$$

and let $u_{k}=y_{2 j}$ when $k=n_{2 j-1}$, and zero otherwise; then $z_{k}=x_{k}+u_{k}$ is a block perturbation of $\left(x_{k}\right)$. By assumption, $\lim _{k \rightarrow \infty} x_{k}=0$ weakly and $\lim _{k \rightarrow \infty} z_{k}=0$ weakly, so that $\lim _{k \rightarrow \infty} u_{k}=0$ weakly; this is a contradiction.

5. Reflexivity. Most of the results of this section remain valid if stated with the condition "every strongly bounded sequence in $E^{\prime \prime}$ is equicontinuous" replacing the condition " $E$ is quasi-barrelled" (or, if $E$ is complete, "barrelled"); however, as shown in section 2, this is only a very slight difference, and in any case this form of the results can be deduced from the weaker form. Thus I shall, where applicable, assume that $E$ is quasi-barrelled or barrelled. It should also be observed that in [4], it is shown that a sequentially complete barrelled space with a Schauder basis is complete, and the results are given for complete barrelled spaces, rather than sequentially complete barrelled spaces.

THEOREM 5.1. Let $E$ be a quasi-barrelled space with a simple normalised Schauder basis $\left(x_{n}\right)$; suppose every simple normalised Schauder basis of $E$ is semi- $\gamma$-complete. Then $\left(f_{n}\right)$ is a shrinking basis of $H$, and if $E$ is sequentially complete (and hence complete), then $\left(x_{n}\right)$ is $\gamma$-complete.

As $\left(x_{n}\right)$ is simple, $\left(f_{n}\right)$ is an equi-Schauder basis of $H$, and in particular is a simple basis of $H$; by Proposition 4.3, $\left(f_{n}\right)$ is semi-shrinking and so is regular. Let $\left(g_{n}\right)$ be any block perturbation of $\left(f_{n}\right)$; and let $\left(y_{n}\right)$ be its dual sequence in $H^{\prime}$; identifying $E$ as a subspace of $H^{\prime}$, it is clear that lin $\left(y_{n}\right)=\operatorname{lin}\left(x_{n}\right)$, and so, as $\left(g_{n}\right)$ is a simple basis of $H,\left(y_{n}\right)$ is a Schauder basis of $E$ in the topology $\beta\left(H^{\prime}, H\right)$. However, by Proposition 5.3 of [5], this is the original topology. As $\left(x_{n}\right)$ is simple and regular, $\left(f_{n}\right)$ is equicontinuous (Lemma 1.2); as $\left(g_{n}-f_{n}\right)$ is strongly bounded, $\left(g_{n}\right)$ is equicontinuous and so $\left(y_{n}\right)$ is normalised.

Suppose that

$$
g_{n}=f_{n}+\sum_{k=1}^{\infty} \delta_{n p_{k}} h_{k},
$$

where $h_{k}=\sum_{m_{k}+1}^{m_{k} k} \alpha_{i} f_{i}, m_{k-1}<p_{k} \leqslant m_{k}$ and $\alpha_{p_{k}}=0$. Suppose, as in Lemma 4.4, $m_{r-1}<\stackrel{m_{k-1}+1}{N} \leqslant m_{r}$ and that $\beta_{N}=0$ if $N<p_{r}$ and $\beta_{N}=1$ if $N \geqslant p_{r}$; then

$$
\sum_{i=1}^{N} f\left(y_{i}\right) g_{i}-\sum_{i=1}^{N} f\left(x_{i}\right) f_{i}=f\left(x_{p_{r}}\right)\left(\beta_{N} h_{r}-P_{N}^{\prime} h_{r}\right)
$$

for all $f \in E^{\prime}$. As $\left(x_{n}\right)$ is bounded, $\sup _{N}\left|f\left(x_{p_{r}}\right)\right|<\infty$; as $\left(h_{r}\right)$ is strongly bounded and $\left(P_{N}^{\prime}\right)_{N=1}^{\infty}$ is strongly equicontinuous, $\left(\beta_{N} h_{r}-P_{N}^{\prime} h_{r}\right)$ is strongly bounded. It follows that $\left(y_{n}\right)$ is a simple normalised basis of $E$.

Therefore $\left(y_{n}\right)$ is semi- $\gamma$-complete; by Proposition 4.3, $\left(g_{n}\right)$ is semishrinking for $H$; applying Theorem 4.5, $\left(f_{n}\right)$ is a shrinking basis of $H$. If $E$ is sequentially complete, then by Corollary 2 of Proposition 5.3 of [5], $\left(x_{n}\right)$ is $\gamma$-complete. 


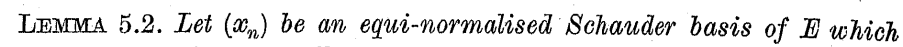
is not semi- $\gamma$-complete; then $E$ has a normalised Schauder basis $\left(y_{n}\right)$ which is not semi-shrinking, and the dual sequence $\left(g_{n}\right)$ may be extended by one element $g_{0}$ to a Schauder basis of $H$.

Suppose $\left(\alpha_{n}\right)$ is a sequence such that $\alpha_{n} \rightarrow 0$ but $\left(\sum_{i=1}^{n} \alpha_{i} x_{i} ; n=1,2, \ldots\right)$ is bounded. Then there exists an increasing sequence $p_{k}$ and $\varepsilon>0$ such that $\left|\alpha_{p_{k}}\right|>\varepsilon$ for all $k$. Let $p_{0}=0$, and

$$
\begin{gathered}
u_{j}=\sum_{p_{j-1}+1}^{p_{j}-1} \alpha_{i} x_{i}, \quad z_{p_{j}}=a_{p_{j}} x_{p_{j}}+u_{j}, \\
z_{n}=x_{n} \quad \text { for } n \neq p_{j} ;
\end{gathered}
$$

then $\left(z_{n}\right)$ is a block perturbation of the Schauder basis $\left(\gamma_{n} x_{n}\right)$, where $\gamma_{p_{j}}=\alpha_{p_{j}}$ and $\gamma_{n}=1$ if $n \neq p_{j}$. Therefore $\left(z_{n}\right)$ is a simple equi-normalised basis of $E$; obviously

$$
\sum_{i=1}^{n} z_{p_{j}}=\sum_{i=1}^{p_{n}} \alpha_{i} x_{i}
$$
is a bounded sequence, and so $\left(z_{p_{j}}\right)$ is a subsequence of type $P$. Applying
Theorem 3.1 , if

$$
y_{p_{n}}=\sum_{i=1}^{n} z_{p_{i}} \quad \text { and } \quad y_{i}=z_{i}\left(i \neq p_{n}\right)
$$

then $\left(y_{n}\right)$ is a normalised Schauder basis of $E$ with $\left(y_{p_{n}}\right)$ a subsequence of type $P^{*}$. If $\left(h_{n}\right)$ is the dual sequence of $\left(z_{n}\right)$, then $h_{p_{1}}\left(y_{p_{n}}\right)=1$ for all $n$, and so $\left(y_{n}\right)$ is not semi-shrinking.

If $\left(g_{n}\right)$ is the dual sequence of $\left(y_{n}\right)$, then

$$
\sum_{i=1}^{n} g_{i}(x) y_{i}-\sum_{i=1}^{n} h_{i}(x) z_{i}=h_{p_{k+1}}(x) y_{p_{k}},
$$

where $\boldsymbol{p}_{k} \leqslant n<p_{k+1}$. As $\left(x_{n}\right)$ is equi-regular, so is $\left(\gamma_{n} x_{n}\right)$ and hence $\left(z_{n}\right)$; thus if $A$ is a bounded subset of $E,\left\{h_{p_{k+1}}(x) y_{p_{k}} ; x \in A, k=1,2, \ldots\right\}$ is also bounded. Therefore $\left\{\sum_{i=1}^{n} g_{i}(x) y_{i} ; x \in A, n=1,2, \ldots\right\}$ is bounded, and
$\left(y_{n}\right)$ is simple.

Thụs $\left(g_{n}\right)$ is a Schauder basis for $\overline{\operatorname{lin}}\left(g_{n}\right)$ in $\mathbb{E}^{\prime}$; letting $g_{0}=h_{p_{1}}$, then lin $\left(g_{i} ; i=0,1,2, \ldots\right)=\operatorname{lin}\left(h_{i}\right)=\operatorname{lin}\left(f_{i}\right)$. If $h_{p_{1}} \epsilon \overline{\operatorname{lin}}\left(g_{i} ; i=1,2, \ldots\right)$,

$$
h_{p_{1}}=\sum_{i=1}^{\infty} g_{p_{i}} \quad \text { in } \beta\left(E^{\prime}, E\right)
$$

but this is impossible as $\left(g_{n}\right)$ is regular; thus $H=\operatorname{lin}\left(g_{0}\right) \oplus \overline{\operatorname{lin}}\left(g_{i} ; i=1,2\right.$, ...) and $\left(g_{i} ; i=0,1,2, \ldots\right)$ is a Schauder basis of $H$.

THEOREM 5.3. Let $E$ be a complete barrelled space with a normalised Schauder basis. The following are equivalent:

(i) Every normalised Schauder basis of $E$ is shrinking.

(ii) Every normalised Schauder basis of $E$ is semi-shrinking.

(iii) Every normalised Schauder basis of $E$ is $\gamma$-complete.

(iv) Every normalised Schauder basis of $E$ is semi- $\gamma$-complete.

(v) $E$ is reflexive.

By Theorem 5.1, (iii) and (iv) are equivalent; while by Theorem 4.5, (i) and (ii) are equivalent. By Lemma 5.2, (ii) implies (iv), and thus (i) implies (iii); thus by Theorem 3.3 of [5], (i) and (v) are equivalent. The proof is completed by showing that (iii) implies (ii).

Suppose $\left(x_{n}\right)$ is a normalised Schauder basis of $E$; then $\left(x_{n}\right)$ is $\gamma$-complete, and by Theorem 6.3 of [5], $H$ is barrelled with $H^{\prime}=E$. Suppose $\left(f_{n}\right)$ is not semi- $\gamma$-complete as a basis of $H$; then $H$ has a normalised Schauder basis $\left(g_{n}\right)$ which is not semi-shrinking, and has a subsequence. $\left(y_{p_{n}}\right)$ of type $P^{*}$. The dual sequence $\left(y_{n}\right)$ of $\left(g_{n}\right)$ may be extended to a basis of $\operatorname{lin}\left(x_{n}\right)$ in $\{E, \beta(E, H)\}$ (Lemma 5.2 ); however, $\beta(E, H)$ is the original topology on $E$ (Proposition 5.3 of [5]), and so $\left(y_{n}\right)$ may be extended by one element $y_{0}$ to a Schauder basis of $E$. As $\left(g_{n}\right)$ is normalised, so is $\left(y_{n}\right)$ (Theorem 3.4 of [6]), and hence so is its extension by one element. Thus $\left(y_{n}\right)$ is semi- $\gamma$-complete; however $\left(\sum_{i=1}^{n} y_{p_{j}}\right)_{n=1}^{\infty}$ is bounded, and so this is
a contradiction.

It follows that $\left(f_{n}\right)$ is a semi- $\gamma$-complete basis of $B$, and so, by Proposition 4.3, $\left(x_{n}\right)$ is semi-shrinking. Thus (iii) implies (ii), and all five conditions are equivalent.

In the case when $E$ is a Banach space, the equivalence of (i), (iii) and (v) was proved by Zippin [12]. This theorem raises the point of whether the same result is true without the restriction of considering only normalised bases. In particular, one might consider whether (iv)

can be replaced by then $\lim \alpha_{n} x_{n}=0 "$

$\lim _{n \rightarrow \infty}$

\section{References}

[1] E. Dubinsky and J. R. Retherford, Schauder bases and Köthe sequence spaces, Trans. Amer. Math. Soc. 130 (1968), p. 265-280.

[2] D. J. H. Garling, On topological sequence spaces, Proc. Camb. Philos. Soc. 63 (1967), p. $963-981$. 
[3] A. Grothendieck, Espaces vectoriels topologiques, Sao Paulo 1954.

[4] N. J. Kalton, Schauder decompositions and completeness, Bulletin of London Math. Soc. 2 (1970), p. 34-36.

[5] - Schauder decompositions of locally convex spaces, to be published in Proc. Cambridge Phil. Soc. [6] - Normalisation properties of Schauder bases, to be published in Proc. London
Math. Soc.

[7] A. Pełczyński and I. Singer, On non-equivalent and conditional bases in Banach spaces, Stud. Math. 25 (1964), p. 5-25.

[8] A. Pełczyński and W. Szlenk, An example of a non-shrinking basis, Rev. Roumaine Math. Pures et Appl. 10 (1965), p. 961 - 966.

[9] J. R. Retherford, Bases, basic sequences and reflexivity of linear topological spaces, Math. Ann. 248 (1966), p. $280-285$.

[10] - A semi-shrinking basis which is not shrinking, Proc. Amer. Math. Soc. 19 (1968), p. 766.

[11] I. Singer, Basic sequences and reflexivity of Banach spaces, Stud. Math. 21 (1962), p. $351-369$.

[12] M. Zippin, A remark on bases and reflexivity in Banach spaces, Israel Jour. Math. 6 (1968), p. 74-80.

[13] T. A. Cook, Schauder decompositions and semi-reflexive spaces, Math. Ann. 182 (1969), p. $232-235$

\section{On non-isomorphism of Banach spaces of holomorphic functions}

by

\section{G. M. HENKIN (Moseow)}

1. The results discussed in this paper are based on two classical theorems of functional analysis. The first of these theorems says that every separable Banach space $X$ is linearly isometric to a subspace of $C=C([0,1])$, the space of continuous functions on the segment. $[0,1]$ (see Banach [1], p. 163). The second theorem, stated in a form which is convenient for our purposes, is the following:

Suppose that the space $C$ is a closed linear subspace of a Banach space $B$. Then there exists a continuous linear operator of extending continuous linear functionals on $C$ to the functionals on $B$ (see Nachbin [4], Lindenstrauss [3], 86-89, and also [8], p. 49).

These results have given to A. A. Miliutin and A. Pełczyński (separately) the idea of trying to establish non-isomorphism of given Banach spaces $X$ and $Y$ by comparing the positions in which these spaces can be embedded into the universal space $C$. The realisation of this idea has supplied the proof of non-isomorphism of certain Banach spaces which could not as yet be distinguished by any other method.

2. Let $X$ be a Banach space and let $J: X \rightarrow C$ be an operator of an isomorphic embedding of $X$ into $C$. Then the conjugate $J^{*}: O^{*} \rightarrow X^{*}$ is onto $X^{*}$. For every subspace $M$ of $X^{*}$, let $\chi_{J}(M)$ denote the infimum of the norms of linear operators $S: M \rightarrow C^{*}$ having the property that $J^{*} S: M \rightarrow M$ is the identity on $M$.

In 1964 A. A. Miliutin comunicated to the author the following (unpublished) result:

THEOREM 1. Let $I: X \rightarrow C$ and $J: X \rightarrow C$ be two isomorphic embeddings of the space $X$ into $G$. Then, for every subspace $M \subset X^{*}$, we have

$$
\left(\|J\| \cdot\left\|I^{-1}\right\|\right)^{-1} \leqslant \chi_{J}(M) / \chi_{I}(M) \leqslant\|I\| \cdot\left\|J^{-1}\right\|,
$$

